\title{
Analysis on the Treatments on Early Diagnosis of Pancreatic Cancer (PC)
}

\author{
Jianing Zhang ${ }^{1 *}$ \\ ${ }^{1}$ Experimental High School Attached To Beijing Normal University, 100044 Beijing, China
}

\begin{abstract}
Pancreatic cancer is one of the deadliest cancer diseases with a relatively high mortality rate. The higher mortality rate of pancreatic cancer is normally a result of late diagnosis. Early diagnosis and detection are quintessential to the survival of pancreatic cancer patients. However, diagnosis of pancreatic cancer at early stages is extremely challenging and difficult by the use of available biomarkers and serological markers. Sometimes this could be due to the fact that pancreatic cancer might not be responsible for signs and symptoms and consequently, the patients could ignore and might not seek medical attention until cancer develops and spreads to other surrounding organs. This late diagnosis of cancer in advanced stages is usually the major cause of a negative prognosis. This paper seeks to critically analyze the treatment on the early diagnosis of pancreatic cancer and the advancements of diagnostic tools and therapies. The conclusion of this paper is that advances in biomarkers for early diagnosis can greatly help patients with pancreatic cancer. In particular, the marker CA19-9, due to its high level of expression in human malignant, benign reactive and metaplastic pancreatic ducts, plays a very important role in the early diagnosis and monitoring of pancreatic cancer.
\end{abstract}

\section{Introduction}

Pancreatic cancer has a relatively high mortality rate but with a low rate of incidence. It is among the leading causes of cancer deaths in the world especially if early detection, diagnosis as well as treatment is not appropriately done [8]. The deficiency of early diagnostic, sophisticated pathophysiology, unresponsiveness to chemotherapy and radiations, and absence of prognostic markers are among the main impediments that hamper successful therapy. The major characteristics of pancreatic cancer are the late-inception symptoms and signs with rapid progression and death. The diagnosis of cancer at the late stages could be due to the absence of clinical signs and symptoms as well as a decreased rate of incidence of this kind of cancerous neoplasia.

Pancreatic cancer falls under the category of diseases that includes endocrine cancer and exocrine pancreases. In this category of diseases, pancreatic cancer accounts for over 90 percent of all cases. Studies have shown that there is evidence from the data obtained from contemporary genomic sequencing of metastatic and primary cases which insinuate that pancreatic cancer tends to slowly develop over a relatively long period of time, taking an estimated average of 17 years to progress to the development of metastatic subclones from the tumor- originating cells and subsequently followed by the death of the patient after about 2.7 years [9]
Research concerning the timeline development of pancreatic cancer reveals that cancer could either be imperceptible or fully resectable. This implies that pancreatic cancer can be resectable approximately six months before diagnosis. Therefore, the slower rate of development of pancreatic cancer together with the improved therapeutic and treatment response of patients who have early-stage pancreatic cancer signifies that early detection and efficient and accurate diagnostic markers could have positive significant impacts on the patients' results or outcomes. However, there are various barriers to the diagnosis and treatment of pancreatic cancer. While early detection and diagnosis of pancreatic cancer have been the most common challenges, the development of biomarkers to help in the early diagnosis of pancreatic cancer has also been one of the major problems in pursuing effortless, efficient, and accurate detection of cancer [7]

This paper critically focuses on the pathological basis of pancreatic cancer and its present diagnostic approaches. In the analysis of the treatment of early diagnosis of pancreatic cancer, advancements of investigation methods and diagnostic tools are very essential.

\section{The Pathological Basis of Pancreatic Cancer}

The pancreases have a neoplasm that consists of a wide spectrum and it is normally categorized based on their

\footnotetext{
* Corresponding author: martinn_zhang@163.com
} 
histological variations. It is classified as either epithelial or non-epithelial. The neoplasm spectrum can also be classified according to their biological behaviors in the malignant, benign as well as pr-malignant neoplasm. Epithelial neoplasm is classified as exocrine and endocrine. The exocrine group can be further categorized into acinar and ductal neoplasms. Pancreatic ductal adenocarcinoma (PDAC) commonly referred to as pancreatic cancer is the most common pancreatic malignancy [14].

PDAC is normally situated in the proximal pancreas. Pancreatic body or tail involvement is normally very rare. In pancreatic head PDAC, any kind of obstruction of the shared bile duct could result in painless jaundice [18]. Nevertheless, diagnosis of pancreatic cancer at an early stage is very difficult. During diagnosis, the PDAC is normally approximately two to four centimeters and sometimes it could be larger especially if it is located in the distal pancreas and had begun to infiltered the surrounding structures such as the duodenum, peripancreatic adipose tissue, and the portal vein among other structures. During diagnosis, the regional lymph node metastases are also normally present. The PDAC is usually presenting itself as a firm and solid whiteyellowish scantily delineated mass. The PDAC comprises of tubular glands which resemble smaller or sometimes medium-sized pancreatic duct. Within the tumor, the patterns of growth are usually heterogeneous. Also, PDAC may include non-tubular elements such as gyriform, clear cell, or cribriform components that could have an impact on the survival of the patients. The PDAC's asymmetrical tumor glands are mostly entrenched in a prominent desmoplastic stroma that comprises extracellular matrix proteins, stroma cells, and inflammatory cells and they are responsible for the neoplasm biological behavior [23]

The PDAC specimens grossing is essential for PDAC case workup. There are three major aspects of the extent of the primary tumor that is very pertinent for the category $\mathrm{T}$ of the staging of the TNM. These aspects include the presence as well as the number of lymph node metastases and also the association of the tumor to resection margins. For the case of pancreaticoduodenectomy specimens, the recommendation of standard slicing techniques of sampling with the specimen done axial sectioning that is perpendicular to a longitudinal axis of the descending duodenum is usually necessary. This is majorly important for the correlation of the MRI imaging or the CT imaging with the microscopic findings [17]. It is also essential for the circumferential resection margin (CRM) evaluation in a microscopic manner and consequently evaluation on the microscopic slide. The CRM encompasses the medial, anterior, and posterior surfaces of the pancreas. These surfaces must be connected with various colors before partitioning based on the predefined color. This is crucial in ensuring that the association of the tumor to each surface can be recapitulated on the microscopic specimens. The CRM is normally influenced by the (R1) resection that is microscopically incomplete and thus, evaluation is always necessary. On the other hand, inking of the anterior and posterior surfaces as well as slicing the specimen perpendicular to the longitudinal axis is commended for the distal pancreatectomy specimen so that the CRM can be evaluated [4]. Therefore, the approach of standardized grossing is very crucial since it aids in the reclamation of a larger number of lymph nodes than the ones in the protocol of the nonstandardized approaches. Standardized grossing approaches are also a germane aspect taking into account the prognostic role of lymph node ratio (LNR).

There are different morphological variations of PDAC. Most of these morphological variants have similar molecular pathogenesis and thus, they have a common prognosis with the typical PDAC and biological behaviors. However, some morphological variants of PDAC have diverse prognoses as well as varied molecular backgrounds. The PDAC variants that have common molecular pathogenesis include the following: undifferentiated or anaplastic carcinoma, micropapillary carcinoma, adenosquamous carcinoma, undifferentiated carcinoma that have giant osteoclastic cells, large-duct carcinoma, and the signet-ring cell carcinoma of the pancreas. The PDAC variants with different molecular pathogenesis on the other hand include the following: medullary carcinoma, hepatoid adenocarcinoma, and the colloid carcinoma of the pancreas [21].

The anaplastic or undifferentiated carcinoma has a poorer prognosis as compared to the classical PDAC. Anaplastic carcinoma is normally characterized by a growth that is dispersed and sometimes a solid growth as well as the presence of huge, conspicuously polymorphous tumor cells and also multinuclear tumor giant cells. On the other hand, adenosquamous carcinoma of the pancreas is a carcinoma that has a squamous element and it makes up over 35 percent of the mass of the tumor[10]. This carcinoma usually has very low glandular elements. Even though adenosquamous carcinoma shares similar carcinogenesis with the classical PDAC, its prognosis is very worse. Unlike anaplastic carcinoma, undifferentiated carcinoma that has osteoclastic giant cells are tumors that have giant histiocytic cells and have a better prognosis. The largeduct carcinoma which normally forms large ducts and sometimes forms dilated ducts can mirror the cystic tumors of the pancreas that are non-invasive. This variant of PDC has common patient survival with typical PDAC. The micropapillary carcinomas are similar to those of breast cancer. They comprise the micropapillary cell clusters that are densely packed within the cleft. These carcinomas have an inside-out pattern of the MUCI staining. The micropapillary carcinoma also includes the cytoplasmic positivity for the galectin as well as ecadherin [21]. The signet-cell carcinomas are normally extremely rare. These carcinomas of the pancreas are characterized by large huge cytoplasmic mucin vacuoles which push the nucleus to the periphery of the cells [10].

Medullary carcinomas of the pancreas usually have diverse patterns of syncytial growth and it shows the pushing inversion. The medullary carcinomas are sometimes linked with the microsatellite instability that is similar to colorectal counterparts and it normally includes necrosis areas. While hepatoid carcinoma is 
extremely rare and is an emulation of hepatocellular carcinoma concerning its immune profile, and morphology, colloid carcinomas of the pancreas are commonly linked with high-grade intestinal-type intraductal papillary neoplasm of the pancreas. The colloid carcinomas are typified by the manifestation of the aggregate of extracellular mucin and approximately 50 percent of these tumors unveil a better prognosis compared to typical PDAC [18].

Research on pancreatic cancer specimens has revealed that most of the pancreatic carcinomas consist of the cells from the ducts. This implies that pancreatic cancer originates from the ductal epithelial cells [2]. These revelations can also be backed up by evidence from the analysis of biochemical phenotype and ultrastructural analysis which also showed that the pancreatic cancer cells had ductal epithelial characteristics. However, the conclusion that the cell that brings about PDAC originates from the ductal epithelial has been strongly challenged. There have been many experiments that have led to arguments about the origin of the PDAC with others arguing that acinar cells could transform into ductal adenocarcinoma. Therefore, with many uncertainties surrounding the origin of PDAC, it is very difficult and challenging in comprehending the true origin of PDAC.

Pancreatic cancer is caused by the mutation of cells in their DNA in the pancreas. The DNA is the most vital component of the cell which controls the cells. The mutation normally leads to the uncontrollable growth of cells and these mutated cells could lead to the death of other normal cells for their survival and continued existence [20]. The mutated cells grow and accumulate forming a tumor which if left untreated, cancer may spread to the surrounding organs such as the duodenum, stomach, portal veins, and even to the distant organs.

\section{The severity of Pancreatic Cancer}

Pancreatic cancer is a very deadly disease with a very high rate of mortality. The pancreatic cancer's particular signs and symptoms in most cases envisage hasty death. This is because PDAC usually develops very quickly once the patient becomes symptomatic [15]. According to the researches, PDAC diagnosed a median of approximately two months after the signs and symptoms onset and death could occur with an estimate of four to six months after diagnosis. The high mortality rate of pancreatic cancer is because Pancreatic cancer is seldomly detected or diagnosed at its early stages when it can easily be cured since it usually does not cause signs and symptoms until after it has spread to the surrounding organs as well as to the distant organs [24]

The preliminary presentation of the PC patients varies with respect to the location of the tumor. It is estimated that about 60 to 70 percent of the PC cases, the tumor is at the head of the pancreas and about 20 to 25 percent of the case, the tumor is located at the tail or body of the pancreas [3]. The main signs and symptoms reported in the patients with PC include the following: abdominal pain, vomiting, asthenia, diarrhea, ascites, jaundice,
Courvoisier's signs, nausea, weight loss, back pain, thrombophlebitis, cachexia, dark urine, anorexia, hepatomegaly, steatorrhea, epigastric pain and mass among others.

Poor prognosis and late presentation are the most common features of PC and are usually elucidated by the stage-specific survival curves for the PC that is histologically confirmed. Prognosis, as well as treatment, usually depends on the stage of the PC at diagnosis. Correct staging is very crucial. Based on the practical staging system, PC patients can be categorized into resectable, unresectable, and borderline resectable. The resectable cancer patients include patients who are at stage 1 and stage 2 of the PC. The stage 3 PC can be treated through radiation and chemotherapy is considered as the only treatment for stage 4 PC [11]. Patients that do not have distant metastases but with the involvement of bold vessels are usually deemed as borderline resectable. Neoadjuvant therapy is an efficient treatment for borderline patients.

Unresectable cancer patients are normally those patients who have stage 4 PC. PC is known to have a dense desmoplastic reaction that normally fosters resistance to chemotherapy. Nevertheless, chemotherapy is the most common option and it is comprehensively used to treat the unresectable PC. Radiotherapy has also been used to treat the unresectable PC even though there is very little evidence to support its efficacy. For unresectable locally progressive tumors. Radiotherapy is used as a palliative treatment option since it can kill cancer cells and inhibit them from growing and recuring [6].

\section{Current Therapies/ Diagnostic approaches on Pancreatic Cancer}

PC is rarely detected at its early stages since it does not normally cause signs and symptoms until after it spreads to other nearby organs as well as distant organs. Therefore, PC is usually not diagnosed until the precipitous advent of prominent clinical signs and symptoms for advanced cancer. Approximately 10 to 20 percent of the PC cases are either resectable or borderline resectable. Therefore, they are suitable for surgery linked with adjuvant or neoadjuvant treatment to cure cancer. However, approximately 80 percent of PC patients are not candidates for hypothetically therapeutic resection [3].

PDAC patients with metastatic disease or advanced tumors do not have any curative option. Current advances in radiotherapy and chemotherapy have led to acceptable advancements in survival in patients who have resectable PC as well as unresectable PC. Other new therapies including gene therapy, anti-angiogenesis treatment, and immunotherapy show good prospects in the future even though they are not yet proven.

Radiotherapy is commonly known for the palliative treatment of patients with unresectable locally advanced tumors. Radiotherapy kills the cancer cells and prevents them from growing and recuring. However, this kind of therapy has side effects such as skin rashes, fatigue, 
toxicity to the nearby normal tissues, and gastrointestinal symptoms [22]. The current advances in radiotherapy such as intensity-modulated radiotherapy (IMRT) and image-guided radiotherapy (IGRT) give an alternative option for treatment that is more effective. Other highly advanced technologies in radiotherapy such as stereotactic body radiotherapy (SBRT) have been deemed to produce accurate radiation dose while ensuring minimizing damage to the nearby normal tissues [6].

Chemotherapy on the other hand is mainly used widely in treating unresectable PC. However, sometimes chemotherapy seems inefficient due to the resilience of PC which is promoted by the dense desmoplastic reaction. Chemotherapy such as GEM/capecitabine, FOLFIRINOX, oxaliplatin (XELOX), GEM/erlotinib, and GEM/NAB-paclitaxel have been used over the years and they play a central role in the adjuvant situation for metastasis PC patients despite having constrained efficacy in metastasis [16].

Surgical resection is normally considered as the only probable way of curing PC patients. Nevertheless, it is estimated that only 20 percent of the patients can have operable PC. The criteria for selecting the appropriate operative procedure for PC depends on various factors such as tumor size, tumor stage, and tumor location [1]. The most popular operative procedure for PC is the classic Whipple procedure which is often referred to as pancreatoduodenectomy usually involves getting rid of the pancreatic head and the curve duodenum, the common bile duct as well as the gallbladder. Distal pancreatectomy is generally recommended for the PC that involves the tail and the body.

\section{Advancement of Diagnosis/Therapies}

Early detection and diagnosis of $\mathrm{PC}$ is the major challenge in managing this disease. Therefore, one of the major targets particularly in the management of $\mathrm{PC}$ is early detection and diagnosis. Current advancement of diagnosis is the key to managing the PC. There are two major divisions of methods that can aid in the early diagnosis of PC. These methods include Biomarkers and imaging methods. The imaging methods involve triflingly invasive or non-invasive screening methods like blood tests for early diagnosis. In this section, the focus is on the biomarker's methods and mainly the Carbohydrate antigen 19-9 (CA19-9).

\section{Carbohydrate antigen (CA19-9)}

The carbohydrate antigen (CA19-9) is one of the biomarkers that has been routinely used to manage PC. However, the CA19-9 has a higher prognosis role substantiating to be positive about 57.1 percent of the PC patients who are Stage 1 and 44.1 percent of PC patients with stage $2 . S$ therefore, it can be applied in suspected cancer or pancreas lesions patients that are identified by imaging methods or patients exhibiting PC symptoms such as obstructive jaundice. The CA19-9 is very essential in monitoring the recurrence of tumors after treatment [12].

The CA19-9 is a variety of biomarker that helps in diagnosing and monitoring the progress of PC. This is possible because the patients that have PC normally have increased blood levels of CA19-9 (a carbohydrate antigen that is found on numerous proteins) healthy people normally have very low levels of CA19-9 in their blood. While high levels of CA19-9 in the blood is normally a sign of PC, it can also indicate other types of cancer as well as noncancerous disorders such as liver cirrhosis and gallstones [5].

To determine the efficacy of the CA19-9 biomarker, an experiment was conducted on the recapitulation of the CA19-9 increase and regulation in cultured mouse PDAC cells as it is observed in human patients. To express CA19-9 in mouse cells, human FUT3 was transduced in the mouse PDAC cells. Although expression of FUT3 only was not adequate for CA19-9 production, it led to elevated levels of Lewis antigens after the terminal galactose moieties that are found in rodents were removed. (Fig. 1A) when the Lewis epitopes are generated, it signifies the reprogramming of the precursor substrates that are required for CA19-9 production in the cells of pancreatic ductal [13]. The expression of both 33 GALT5 and FUT3 in the PDAC cells of a mouse resulted in the cell surfacing expression of CA19-9 at levels that are equivalent to those observed in the cancer cells of human patients (Fig. 1B and S1B). 

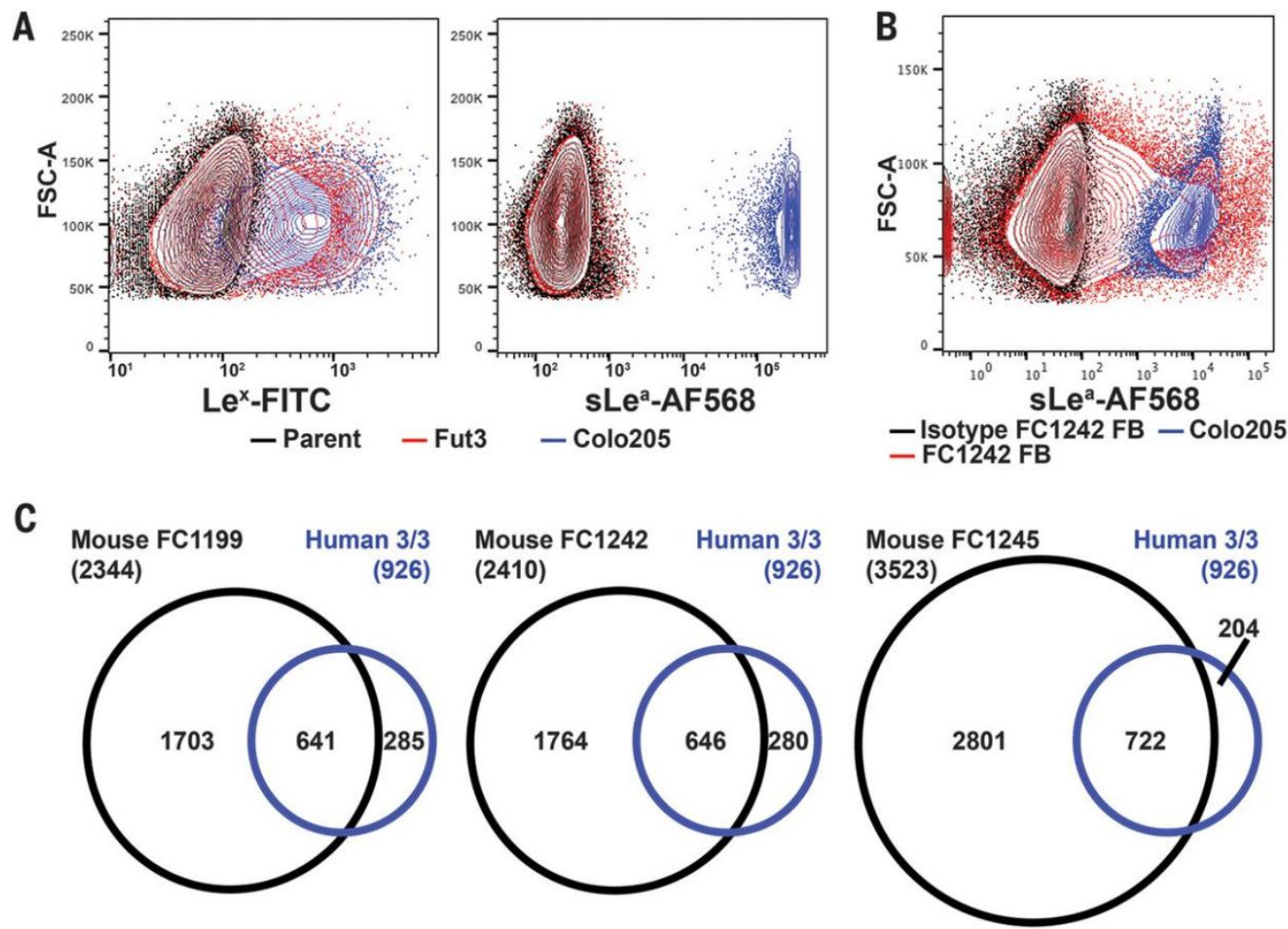

Fig. 1. FUT3 with $\beta 3$ GALT5 expression enables CA19-9 production in engineered mouse pancreatic cancer cells.

(A) Ectopic FUT3 engenders Lewis (Lex) but not CA19-9sLea expression in PDAC cells of mouse by flow cytometric analysis. The Lex- and CA19-9-sLea-positive human cell line COLO205 and Lex- and CA19-9-sLea-negative parental KPC cell lines are shown. FITC, fluorescein isothiocyanate; FSC-A, forward scatter-area; AF568, Alexafluor 568.

(B) CA19-9 flow cytometry of PDAC cells of mouse sturdily and expressing constitutively 33 GALT5 (FB) and FUT3 and contrasted with the isotype control antibody.

(C) Overlap among CA19-9 protein carriers spotted in three out of three PDAC cell lines of humans $(n=926)$ with three individual PDAC cell lines of a mouse expressing 33 GALT5 andFUT3.

The major objective of the experiment was to compare the mouse CA19-9 protein carriers and human CA19-9 protein carriers. The result obtained showed that an approximated average of 72.3 percent $(n=3$ and 95 percent confidence interval) of the CA19-9 bespoke protein spotted in the three PDAC cell lines of human patients was also present in the PDAC cell lines of the cultured murine. Therefore, the expression of humans B3GALT5 and FUT3 genes in cells of a mouse greatly recapitulates the human CA19-9 carrier profile [13].

Detection of CA19-9 expediates quick screening as well as diagnosis of PDAC. Increased levels of CA19-9 in the blood are usually a sign of PDAC. However, high levels of CA19-9 in the blood are also associated with other cancer diseases such as breast cancer, lung cancer, ovarian cancer, throat cancer among other cancer diseases. Elevated levels of CA19-9 are also associated with non-cancerous disorders such as gallstones and cirrhosis [19]. Therefore, CA19-9 can also be used in monitoring the progression of other types of cancer diseases such as breast cancer, lung cancer, ovarian cancer among other cancer diseases.

\section{Conclusion}

Pancreatic cancer is a very belligerent and fatal disease with a very high mortality rate. Early detection and diagnosis of PC is the major challenge in managing cancer. It does not normally cause signs and symptoms until after it has spread to the surrounding organs. Characteristically, PC usually begins by metastasizing to the regional lymph nodes and spread to other nearby organs such as the stomach, duodenum, colon, and liver. PC might also metastasize to surfaces in the abdominal cavity through the peritoneal spread. Patients with PDAC normally experience complications such as gastric outlet obstruction, biliary obstruction, and server abdominal pains.

The origin of $\mathrm{PC}$ is unclear and it remains controversial. While PC might have limited treatment options, advancement in early diagnostic biomarkers with the potential of detecting the disease when it is still in its preinvasive stage can drastically change the patients' fate. Various biomarkers have been tested and used for the detection of PC. The CA19-9 biomarker has been very vital in the early diagnosis and monitoring of the PC. The CA19-9 in the normal pancreatic duct is expressed in low levels while it normally becomes higher in the malignant, benign-reactive, and metaplastic ducts in humans. Advancement in efficient and effective diagnostic and therapy methods is fundamental in the early detection and management of PC. 


\section{Acknowledgements}

First and foremost, I would like to show my deepest gratitude to my teachers and professors in my university, who have provided me with valuable guidance in every stage of the writing of this thesis. Further, I would like to thank all my friends and roommates for their encouragement and support. Without all their enlightening instruction and impressive kindness, I could not have completed this paper.

\section{References}

1. I. Amanam \& V. Chung Targeted therapies for pancreatic cancer. Cancers (2018).

2. American Cancer Society. What Is Pancreatic Cancer? (2016).

3. American Cancer Society. Key Statistics for Pancreatic Cancer (2018).

4. N. Bardeesy \& R. A. DePinho. Pancreatic cancer biology and genetics. Nature Reviews Cancer,(2002).

5. T. M. Bauer, B. F. El-Rayes, X. Li, N. Hammad, P. A. Philip, A. F. Shields, T. Bekaii-Saab, Carbohydrate antigen 19-9 is a prognostic and predictive biomarker in patients with advanced pancreatic cancer who receive gemcitabinecontaining chemotherapy: A pooled analysis of 6 prospective trials. Cancer (2013). .

6. K. E. Burton, Radiotherapy. In Management of Adult Glioma in Nursing Practice (2019). .

7. Cancer Research UK. Pancreatic cancer statistics Cancer Research UK (2016). .

8. D. Casado, R. V. Tocino, E. Fonseca, B. Cigarral, B. Barrios, E. Escalera, J. J. Cruz, Pancreatic cancer. Medicine (Spain)(2021)

9. Y. Chen, C. Wang, Y. Zhang, Pancreatic cancer. In Occupational Cancers (2020)..

10. E. A. Collisson, P. Bailey, D. K. Chang, A. V. Biankin, Molecular subtypes of pancreatic cancer. Nature Reviews Gastroenterology and Hepatology (2019)..

11. T. Conroy, F. Desseigne, M.Ychou, O. Bouché, R. Guimbaud, Y. Bécouarn, M. Ducreux, FOLFIRINOX versus Gemcitabine for Metastatic Pancreatic Cancer. New England Journal of Medicine (2011).

12. J. C. Gui,W. L. Yan,X. D. Liu, CA19-9 and CA242 as tumor markers for the diagnosis of pancreatic cancer: a meta-analysis. Clinical and Experimental Medicine (2014).

13. H. Villanueva, E. Hijona, A. Cosme, L. Bujanda, Mouse models of pancreatic cancer. World Journal of Gastroenterology (2012).

14. M. Hidalgo, Pancreatic Cancer: Pancreatic Cancer: Overview. Lancet (2011)..

15. M. Ilic, \& I. Ilic, Epidemiology of pancreatic cancer. World Journal of Gastroenterology (2016).
16. Januszewski, A., \& Popat, S. (2015). Chemotherapy. ERS Monograph.

17. Li, C., Heidt, D. G., Dalerba, P., Burant, C. F., Zhang, L., Adsay, V., ... Simeone, D. M. (2007). Identification of pancreatic cancer stem cells. Cancer Research.

18. Maitra, A., \& Hruban, R. H. (2008). Pancreatic cancer. Annual Review of Pathology: Mechanisms of Disease.

19. Odagiri, E. (2005). CA19-9. Nippon Rinsho. Japanese Journal of Clinical Medicine.

20. Smith, J. K., Chu, Q. D., \& Tseng, J. F. (2015). Pancreatic adenocarcinoma. In Surgical Oncology a Practical and Comprehensive Approach.

21. UK, C. R. (2014). Types of pancreatic cancer. Cancer Research UK.

22. Van Herk, M. (2004). Errors and Margins in Radiotherapy. Seminars in Radiation Oncology.

23. Vincent, A., Herman, J., Schulick, R., Hruban, R. H., \& Goggins, M. (2011). Pancreatic cancer. The Lancet.

24. Yachida, S., Jones, S., Bozic, I., Antal, T., Leary, R., Fu, B., ... Iacobuzio-Donahue, C. A. (2010). Distant metastasis occurs late during the genetic evolution of pancreatic cancer. Nature. 